Frequency of depression and anxiety among patients with chronic spontaneous urticaria visiting a tertiary care hospital in Karachi, Pakistan

Muhammad Rizwan Rafique

Sadia Masood

Sana Tanzil

Saadia Tabassum

Shaheen Naveed

Follow this and additional works at: https://ecommons.aku.edu/pakistan_fhs_mc_med_intern_med

Part of the Internal Medicine Commons 


\title{
Frequency of depression and anxiety among patients with chronic spontaneous urticaria visiting a tertiary care hospital in Karachi, Pakistan
}

Muhammad Rizwan Rafique', Sadia Masood ${ }^{2}$, Sana Tanzil ${ }^{3}$, Saadia Tabassum4, Shaheen Naveed ${ }^{5}$

\begin{abstract}
Objective: To determine the frequency of anxiety and depression among chronic spontaneous urticaria patients presenting at a tertiary care hospital.

Methods: The prospective, cross-sectional study was conducted at the Aga Khan University Hospital, Karachi, from February to August 2015, and comprised patients diagnosed with chronic spontaneous urticaria at the dermatology clinic. All the participants were assessed for anxiety and depression using Aga Khan University-Anxiety Depression Scale. Data was collected in face-to-face interview using a structured questionnaire. The association of the condition with different characteristics were calculated after stratification for age, gender, occupation and monthly income. SPSS 19 was used for data analysis. Results: Of the 146 study participants, 84(57.5\%) were males and 62(42.5\%) were females. The overall mean age was $39 \pm 15.6$ years. Of the total, $39(26.7 \%)$ had depression and anxiety. Depression and anxiety were more frequent among patients with a positive family history of mental disorders $(p=0.02)$.

Conclusion: Anxiety and depression were found to be considerably common among chronic spontaneous urticaria patients, with patients having a positive family history for mental illnesses particularly vulnerable.
\end{abstract}

Keywords: Chronic spontaneous urticaria, Depression, Anxiety, Pakistan. (JPMA 70:511; 2020).

https://doi.org/10.5455/JPMA.297139

\section{Introduction}

Urticaria is one of the most common skin diseases characterised by itchy wheals with or without angioedema that usually persists for less than 24 hours. ${ }^{1}$ Clinical symptoms are due to the binding of antigen-to-antigen specific imuunoglobulin $E$ (IgE) on mast cells and basophils. ${ }^{2}$ Chronic spontaneous urticaria (CSU) is defined as spontaneous) development without external physical stimuli of wheals, angioedema, or both for duration of more than 6 weeks. 3

The cause cannot be found in $50-75 \%$ cases $^{4}$ and up to $40 \%$ of such cases are associated with autoimmune reaction. ${ }^{5}$ The prevalence of urticaria has been reported in many studies between $0.5-1 \%$, while lifetime prevalence is around $15-20 \% .^{1-5}$ Peak incidence had been reported between 20-40 years of age. Females are reported to suffer CSU nearly twice compared to males. ${ }^{1}$ In $30-55 \%$ of CSU cases, the duration is estimated to be 1-5 years, but in 2,4Aga Khan University Hospital, Karachi, Pakistan; ${ }^{3}$ APPNA Institute of Public Health, Jinnah Sindh Medical University, Karachi, Pakistan; 5 Liaquat National Hospital Karachi, Pakistan.

Correspondence: Sadia Masood. e-mail: sadia.masood@aku.edu some cases it can extend up to 50 years. 1,6

Depression and anxiety are the most common psychiatric disorders associated with CSU. The prevalence of depression and anxiety in CSU patients ranges from 13.5\% to $40 \%$ and $7 \%$ to $30 \%$ respectively. ${ }^{7-11}$ The overall mean prevalence of anxiety and depressive disorders among the general population of Pakistan is reported to be $33.6 \% .{ }^{12}$ Quality of life in patients with CSU is as compromised as in many other chronic skin diseases, like psoriasis, atopic eczema and leprosy, along with increased risk of anxiety, depression and somatoform disorders.9,13,14 The presence of depression and anxiety in CSU compromises the quality of life and can also lead to poor compliance with treatment and could lead to decline in patient's satisfaction with medical care, which results in the worsening of the situation.

CSU is one of the most disabling conditions, but its effect on patient's quality of life is often underestimated, with few reports in literature and no previous study in the local population. The current study was planned to evaluate the prevalence of depression and anxiety in CSU.

Vol. 70, No. 3, March 2020 


\section{Patients and Methods}

The prospective, cross-sectional study was conducted at the Department of Dermatology, Aga Khan University Hospital (AKUH), Karachi, from February to August, 2015. AKUH is a leading tertiary care hospital with wellestablished and equipped Dermatology Department, which receives complicated and referred patients from within the hospital and also from other remote areas of the country.

After approval was taken from the institutional ethics review committee, the sample size was calculated by using World Health Organisation (WHO) software. ${ }^{13}$ The frequency of depression and anxiety among CSU patients was based on earlier studies and ranged 7-40\%.9,14 Therefore, while taking the anticipated proportion of depression and anxiety to $40 \%$, the sample size was worked out at $95 \%$ level of confidence and precision of $8 \%$. Non-probability convenience sampling was used to raise the sample. All adult diagnosed CSU patients aged 14-60 years regardless of gender willing to take part in study were recruited. Patients with diagnosed psychiatric illness or those who were taking any psychiatric medication before CSU onset were excluded, and so were those who were not willing to volunteer.

After getting informed consent, general assessment was done using a structured questionnaire which included sociodemographic characteristics. Depression and anxiety were assessed by using the Aga Khan University Anxiety and Depression Scale (AKUADS) which comprised 25 items; 13 psychological and 12 somatic. It is a validated tool in Urdu language for the local community against a gold standard of assessment. ${ }^{15}$ Total scores range $0-75$, and those scoring 19 or above are considered to be suffering from anxiety and depression. ${ }^{15}$ It is a differential scale rank ordered for severity, and obtains an estimate of the distress caused by the attribute being assessed from the respondent directly on a four-point scale varying from 'present all the time (almost daily)', 'most of the time (2 to 5 times in a week)', 'sometimes only ( 2 or less than 2 times in a week)' and 'not present at all'. It decreases the chances

Table: Association of different characteristics of chronic spontaneous urticaria (CSU) patients with depression and anxiety ( $n=146)$.

\begin{tabular}{|c|c|c|c|c|c|}
\hline Variables & & Frequency n (\%) & Depression and anxiety $\mathrm{n}(\%)$ & No depression and anxiety $n$ (\%) & $p$-value \\
\hline \multirow[t]{3}{*}{ Age } & $\geq 25$ years & $37(25.3)$ & $9(23.1)$ & $28(26.2)$ & 0.25 \\
\hline & $26-50$ years & $71(48.6)$ & $16(41)$ & $55(1.4)$ & \\
\hline & $<51$ years & $38(26)$ & $14(39.9)$ & $24(33.4)$ & \\
\hline \multirow[t]{2}{*}{ Sex } & Male & $84(57.5)$ & $26(66.7)$ & $58(54.2)$ & - \\
\hline & Female & $62(42.5)$ & $13(33.3)$ & $49(45.8)$ & \\
\hline \multirow[t]{3}{*}{ Marital status } & Single & $41(28.1)$ & $9(23.1)$ & $32(29.9)$ & 0.65 \\
\hline & Married & $96(65.8)$ & $28(71.8)$ & $68(63.6)$ & \\
\hline & Widow & $9(6.2)$ & $2(5.1)$ & $7(6.5)$ & \\
\hline \multirow[t]{5}{*}{ Educational status } & Uneducated & $11(7.5)$ & $2(5.1)$ & $9(8.4)$ & 0.45 \\
\hline & Informal education & $15(10.3)$ & $6(15.4)$ & $9(8.4)$ & \\
\hline & Primary education & $40(27.4)$ & $13(33.3)$ & $27(25.2)$ & \\
\hline & Secondary education & $28(7.5)$ & $5(12.8)$ & $23(21.5)$ & \\
\hline & Graduation or above & $52(\%)$ & $13(33.3)$ & $39(36.4)$ & \\
\hline \multirow[t]{3}{*}{ Monthly income (PKR) } & $\geq 25,000$ & $38(26)$ & $13(33.3)$ & $25(23.4)$ & 0.38 \\
\hline & $26,000-50,000$ & 35 & $7(17.9)$ & $28(26.2)$ & \\
\hline & $>50,000$ & $75(\%)$ & $19(48.7)$ & $54(50.5)$ & \\
\hline \multirow[t]{6}{*}{ Occupation } & Self employed & $14(9.6)$ & $5(12.8)$ & $9(8.4)$ & 0.82 \\
\hline & Private job & $51(34.9)$ & $14(35.9)$ & $37(34.6)$ & \\
\hline & Government Job & $9(6.2)$ & $2(5.1)$ & $7(6.5)$ & \\
\hline & House maker & $43(29.5)$ & $12(30.8)$ & $31(29)$ & \\
\hline & Student & $27(18.5)$ & $5(12.8)$ & $22(20.6)$ & \\
\hline & Jobless & $2(1.4)$ & $1(2.6)$ & $1(0.9)$ & \\
\hline \multirow[t]{2}{*}{ History of Angioedema } & Yes & $4(2.7)$ & $2(5.1)$ & $2(1.9)$ & 0.28 \\
\hline & No & $142(97.3)$ & $37(94.9)$ & $105(98.1)$ & \\
\hline \multirow[t]{2}{*}{ Family history of mental disorder } & Yes & & $4(10.3)$ & $2(1.9)$ & 0.02 \\
\hline & No & & $35(89.7)$ & $105(98.1)$ & \\
\hline \multirow[t]{2}{*}{ Urticaria duration } & $<6$ months & 133(91.1) & $35(89.7)$ & $98(91.6)$ & 0.72 \\
\hline & $\geq 6$ months & $13(8.9)$ & $4(10.3)$ & $49(8.4)$ & \\
\hline
\end{tabular}

$p<0.05$ significant, PKR: Pak Rupee 
of inter-rater bias and makes establishing inter-rater reliability relatively easy. ${ }^{15}$

Data was analysed using SPSS 19. Mean and standard deviation (SD) were calculated for continuous variables, whereas frequencies and percentages were calculated for categorical variables, such as gender, occupation, marital status, level of education and household income. Patients scoring $\geq 9$ were labeled as having depression and anxiety. The association of different characteristics were also evaluated after stratification for age, gender, occupation and monthly income, and then compared using chi square or fisher exact test. $\mathrm{P}<0.05$ was considered significant.

\section{Results}

Of the 146 study participants, 84(57.5\%) were males and $62(42.5 \%)$ were females. The overall mean age was $39 \pm 15.6$ years, with $71(48.6 \%)$ between $26-50$ years of age, and $37(25.3 \%)$ were 25 years or less. Majority of the participants were married $96(65.8 \%) ; 52(35.6 \%)$ had education up to graduation or above; 11 (7.5\%) were uneducated; $15(10.3 \%)$ had primary education; $40(27.4 \%)$ had education up to middle or matric levels; 51(34.9\%) were employed in theprivate sector; 43(29.4\%) were household women; and $4(2.7 \%)$ had a positive history of angioedema.

Depression and anxiety was found in 39(26.7\%) patients. There was no statistically significant differences between demographic characteristics of CSU patients who had depression and anxiety compared to CSU patients with no depression or anxiety ( $p>0.05$ ). However, a statistically significant difference was observed in family history of mental disorders or illness between CSU patients with depression and anxiety and those with no depression and anxiety $(p=0.02)$.

Among those who had depression and anxiety, 16(41.0\%) were aged 26-50 years, but the difference in frequency of depression and anxiety between different age groups was not statistically significant $(p=0.25)$.

Among those who had depression and anxiety, 39(89.7\%) participants had a duration of illness $<6$ months, while $4(10.3 \%)$ reported a duration of 6 months or more. Also, $13(33.3 \%)$ of the depressed patients were from the lowincome group; $7(17.9 \%)$ belonged to the middle income group; and 19 (48.7\%) were from the high income group (Table).

\section{Discussion}

The current study is one of the few studies conducted in dermatology clinics to assess anxiety and depression in the local context. The CSU can affect every age group 16,17 but the combined frequency of depression and anxiety estimated was slightly higher. Literature has been reporting high prevalence of mental disorders, like anxiety and depression, among the general population of Pakistan. ${ }^{17}$ However, the reported prevalence of anxiety and depression in the general population vary widely because of difference in study populations and assessment methods. CSU has been reported to affect the mental health with a substantial increase in the burden of anxiety and depression among this vulnerable population.

A high prevalence of psychiatric disorders in CSU patients had been reported in a previous study. It highlighted that the quality of life (QOL) in dermatological patients is a strong predictor for psychiatric comorbidity. ${ }^{18}$ Poon et al. found QOL impairment in CSU patients higher than the two common debilitating dermatoses, acne and vitligo, known to affect patients' QOL significantly. ${ }^{19,20}$ Anxiety and depression was seen regardless of the disease duration. The study results are consistent with previous studies. 20

The current study used the AKUADS to assess the anxiety and depression score of the cases. ${ }^{15}$ This is a very simplified Urdu proforma, which facilitated the assessment in the current study and had a high positive predictive value (PPV) of around $83 \% .{ }^{15}$ This scale has also been utilised in multiple other studies conducted in Pakistan and India. 21,22,23,24 Thus, it can also be considered a useful diagnostic and screening tool to assess psychiatric issues in patients presenting with various dermatological conditions. QOL questionnaires may be suitable in one cultural setting and may not be effective in others, therefore revalidation may be necessary in a new environment. ${ }^{25,26}$ In dermatological patients, the degree of psychiatric comorbidity varies in accordance with the disease state, setting of a study itself and the scale used. ${ }^{22}$ The study findings highlighted the importance of timely detection of psychiatric disorders by dermatologists in patients presenting with CSU with the help of a good screening instrument.

The current study has several inherent limitations. First of all, it was a cross-sectional study so temporality between the components cannot be established. Secondly, the study was conducted in a private and relatively expensive 
health facility and their characteristics might be different from patients at public hospitals. Hence, the study cannot provide actual prevalence or burden of depression and anxiety in the general population suffering from CSU. Thirdly, the study confined itself to CSU patients, and, hence, correlation of depression and anxiety with other types of urticaria cannot be determined.

Multi-center studies are recommended which would give a better understanding of the relationship of risk factors among CSU patients.

\section{Conclusion}

Mental health is a neglected area in many parts of Pakistan. The study highlighted positive family history of mental disorders as a possible and important risk modifier for the development of anxiety and depression among CSU patients.

\section{Disclaimer: None. \\ Conflict of Interest: None. Source of Funding: None.}

\section{References}

1. Maurer M, Weller K, Bindslev-Jensen C, Giménez-Arnau A, Bousquet $\mathrm{PJ}$, Bousquet J, et al. Unmet clinical needs in chronic spontaneous urticaria. A GA ${ }^{2}$ LEN task force report. Allergy 2011;66:317-30.

2. Maurer M, Rosén $K$, Hsieh HJ, Saini S, Grattan C, Gimenéz-Arnau A, et al. Omalizumab for the treatment of chronic idiopathic or spontaneous urticaria. N Engl J Med 2013;368:924-35.

3. Zuberbier T, Maurer M. Urticaria: current opinions about etiology, diagnosis and therapy. Acta Derm Venereol 2007;87:196-205.

4. Greaves MW. Pathophysiology of chronic urticaria. Int Arch Allergy Immunol 2002;127:3-9.

5. Schocket AL. Chronic urticaria: pathophysiology and etiology, or the what and why. Allergy Asthma Proc 2006;27:90-5.

6. Beltrani VS. An overview of chronic urticaria. Clin Rev Allergy Immunol 2002;23:147-69.

7. Aslam R, Qadir A, Asad F. Psychiatric morbidity in dermatological outpatients: an issue to be recognized. J Pak Assoc Derma 2007; 17:235-9.

8. Uguz F, Engin B, Yilmaz E. Axis I and Axis II diagnoses in patients with chronic idiopathic urticaria. J Psychosom Res 2008;64:225-9.

9. Staubach P, Dechene M, Metz M, Magerl M, Siebenhaar F, Weller K, et al. High prevalence of mental disorders and emotional distress in patients with chronic spontaneous urticaria. Acta Derm Venereol 2011;91:557-61.

10. World Health Organization. Sample size determination: a user's manual. Epidemiological and Statistical Methodology Unit. [Online] 1986 [Cited 2015 January 15]. Available from URL: http://www.who.int/iris/handle/10665/61764

11. Ozkan M, Oflaz SB, Kocaman N, Ozseker F, Gelincik A, Büyüköztürk $S$, et al. Psychiatric morbidity and quality of life in patients with chronic idiopathic urticaria. Ann Allergy Asthma Immunol 2007;99:29-33.

12. Mirza I, Jenkins R. Risk factors, prevalence, and treatment of anxiety and depressive disorders in Pakistan: systematic review. BMJ 2004;328:794.

13. Sheehan-Dare RA, Henderson MJ, Cotterill JA. Anxiety and depression in patients with chronic urticaria and generalized pruritus. Br J Dermatol 1990;123:769-74.

14. Fried RG, Gupta MA, Gupta AK. Depression and skin disease. Dermatol Clin 2005;23:657-64.

15. Ali BS, Reza H, Khan MM, Jehan I. Development of an indigenous screening instrument in Pakistan: the Aga Khan University Anxiety and Depression Scale. J Pak Med Assoc 1998;48:261-5.

16. Choi SH, Baek HS. Approaches to the diagnosis and management of chronic urticaria in children. Korean J Pediatr 2015;58:159-64.

17. Khan MZ, Naeem A, Mufti KA. Prevalence of mental health problems in acne patients. J Ayub Med Coll Abbottabad 2001;13:7-8.

18. Picardi A, Abeni D, Melchi CF, Puddu P, Pasquini P. Psychiatric morbidity in dermatological outpatients: an issue to be recognized. Br J Dermatol 2000;143:983-91.

19. Lasek RJ, Chren MM. Acne vulgaris and the quality of life of adult dermatology patients. Arch Dermatol 1998;134:454-8.

20. Poon E, Seed PT, Greaves MW, Kobza-Black A. The extent and nature of disability in different urticarial conditions. Br J Dermatol 1999;140:667-71.

21. Ali BS, Amanullah S. A comparative review of two screening instruments: the Aga Khan University Anxiety and Depression Scale and the self reporting questionnaire. J Pak Med Assoc 1998;48:7982.

22. Shahid M, Rehmani R, Khan MM. Anxiety and depression among young adults with chest pain. J Coll Physicians Surg Pak 2004;14:700.

23. Gupta MA, Gupta AK, Schork NJ, Ellis CN. Depression modulates pruritus perception: a study of pruritus in psoriasis, atopic dermatitis, and chronic idiopathic urticaria. Psychosom Med 1994;56:36-40.

24. Ali BS, Rahbar MH, Naeem S, Tareen AL, Gul A, Samad L. Prevalence of and factors associated with anxiety and depression among women in a lower middle class semi-urban community of Karachi, Pakistan. J Pak Med Assoc 2002;52:513-7.

25. Gurel MS, Yanik M, Simsek Z, Kati M, Karaman A. Quality of life instrument for Turkish people with skin diseases. Int J Dermatol 2005;44:933-8.

26. Morgan M, McCreedy R, Simpson J, Hay RJ. Dermatology quality of life scales--a measure of the impact of skin diseases. Br J Dermatol 1997;136:202-6. 